\title{
DIFFUSION BACKGROUND MODEL FOR MOVING OBJECTS DETECTION
}

\author{
Boris V. Vishnyakov, Sergey V. Sidyakin, Yuri V. Vizilter \\ State Research Institute of Aviation Systems (FGUP GosNIIAS), Moscow, Russia - vishnyakov@gosniias.ru
}

Commission III, WG V/5 and WG III/3

KEY WORDS: Background modelling, movement detection, diffusion maps, regression, LBP descriptor

\begin{abstract}
:
In this paper, we propose a new approach for moving objects detection in video surveillance systems. It is based on construction of the regression diffusion maps for the image sequence. This approach is completely different from the state of the art approaches. We show that the motion analysis method, based on diffusion maps, allows objects that move with different speed or even stop for a short while to be uniformly detected. We show that proposed model is comparable to the most popular modern background models. We also show several ways of speeding up diffusion maps algorithm itself.
\end{abstract}

\section{INTRODUCTION}

Recently a lot of background models intended for moving object detection and foreground segmentation were introduced. Moreover, changedetection.net benchmark was created (Goyette, 2012, Y. Wang, 2014) for testing and ranking existing and new algorithms for change and motion detection.

In the paper (Vishnyakov, 2012) we introduced the regression pseudospectrum background model, that was based on the fast way of accumulating the layers of the spectrum using the regression model. However, despite the very high computation speed, that approach lacked quality in complex conditions (swaying branches, changeable lighting conditions, slow objects)

The idea of our new approach is as follows: we improve regression background model using diffusion maps and diffusion regressive filtering.

Below in this paper we describe our approach and provide evaluation results on the changedetection.net database for the most suitable categories: «baseline», «thermal», «bad weather», «low framerate».

\section{DIFFUSION BACKGROUND MODEL}

\subsection{Diffusion map}

Let $I(k)$ be the input image on the frame $k$. Let us assume that $I(k)$ is a grayscale image (or it has been converted to grayscale), $I(k, q)$ is the brightness value of the image $I$ in pixel $q$.

The basis of our approach is the diffusion morphology (Vizilter, 2013) which allows comparing images by shape matching using the projection of image one on image two. In this morphology the projection is evaluated using diffusion maps, that were introduced in (Lafon, 2004, Coifman, 2006a, Coifman, 2006b):

$$
P_{I_{2}}^{I}(k, p)=\sum_{q} A_{p, q}(k) I_{2}(q)
$$

where $p, q$ - points,

$A_{p, q}(k)=\frac{\exp \left(-\frac{\|v(p)-v(q)\|^{2}}{\varepsilon}\right)}{\sum_{q} \exp \left(-\frac{\|v(p)-v(q)\|^{2}}{\varepsilon}\right)}-$ a diffusion operator (diffusion map),

$v(p)$ - a feature vector, calculated in the point $p$ of the image $I$ with the square kernel, $\|\cdot\|-$ a distance between two feature vectors.

\subsection{Complex LBP Descriptor}

In the papers (Gorbatsevich, 2014, Vishnyakov, 2014) the complex LBP descriptor was introduced as a feature vector $\mathrm{v}(\cdot)$ along with the hamming distance $\|\cdot\|$ as a distance between feature vectors for the diffusion maps. This allows very fast computation of the diffusion map.

Our implementation provides a possibility for real time image processing. The computation of diffusion filtering with heat kernel in its original form is an extremely time-consuming procedure even for reasonable neighbourhood of $p$. We propose to substitute such computationally unpleasant descriptors by the combination of intensity $I(p)$ and threshold LBP (Ahonen, 2004) for $v(p)$. In our experiments, the mean value of intensity in the $\mathrm{p}$ neighbour was used. Mean value is computed by a fast algorithm with sliding sum recalculation (but this is not presented in code below). The local binary pattern (LBP) is calculated as a 64-bit vector for each pixel $p$ based on a comparison of its value and values of its neighbours in sliding window. If the value of neighbour pixel is less than the value of central pixel and the difference between them is greater than threshold, then the corresponding bit is set to 1 , otherwise - to 0 . We substitute the original neighbourhood matching metrics by LBP matching metric - Hamming distance, the mean values of intensities are compared by threshold. As local binary patterns are stored as bit fields, the computation of Hamming distance is performed via bitwise XOR operation. The exponent is calculated using table values. Due to this, the usage of our complex LBP descriptor allows both increasing the computational speed and obtaining heat kernels very similar to original.

\subsection{Background model}

The idea of the paper is as follows: the approach for the image comparison in the diffusion morphology can be also used for the moving object detection. For robustness of the approach we propose regression accumulators (Vishnyakov, 2012) $m_{n}(k)$ and $m_{n}^{*}(k)$ for both the original image and the filtered image respectively:

$$
\begin{gathered}
m_{n}(k)=\alpha \cdot m_{n}(k-1)+(1-\alpha) \cdot I(k), \\
m_{n}^{*}(k)=\alpha \cdot m_{n}^{*}(k-1)+(1-\alpha) \cdot I^{*}(k),
\end{gathered}
$$


where $I^{*}(k)=P_{m_{n}(k)}^{I(k)}(k)$ is a diffusion filter of the $I(k)$, which is the projection of $I(k)$ on the memory $m_{n}(k), n$ is a parameter, related to the memory length, $\alpha=\alpha(n)$.

After computing $m_{n}(k)$ and $m_{n}^{*}(k)$, we compare the difference

$$
D(k)=\left|m_{n}^{*}(k)-I^{*}(k)\right|
$$

to the threshold in each pixel and get the binary moving object mask $M(k)$ :

$$
M(k)=\left\{\begin{array}{l}
1, D(k) \geq t h r \\
0, D(k)<t h r
\end{array}\right.
$$

\subsection{Illustrations}

On figure 1 we show smoothed accumulator (memory) $m_{n}^{*}(k)$ using regression model for the projection image $I^{*}(k)-$ a diffusion filter of the $I(k)$. As you can see, no moving object is present on this accumulator. In addition, the accumulator is smoothed because of smoothing properties of the diffusion map. On figure 2 we show the projection image $I^{*}(k)$ itself. One moving object is present on this image. The projection image is also smoothed.

On figure 3 we show the difference $D(k)$ between projection image $I^{*}(k)$ and smoothed accumulator $m_{n}^{*}(k)$.

On figure 4 we show image $M(k)$ - binarized using threshold difference image $D(k)$.

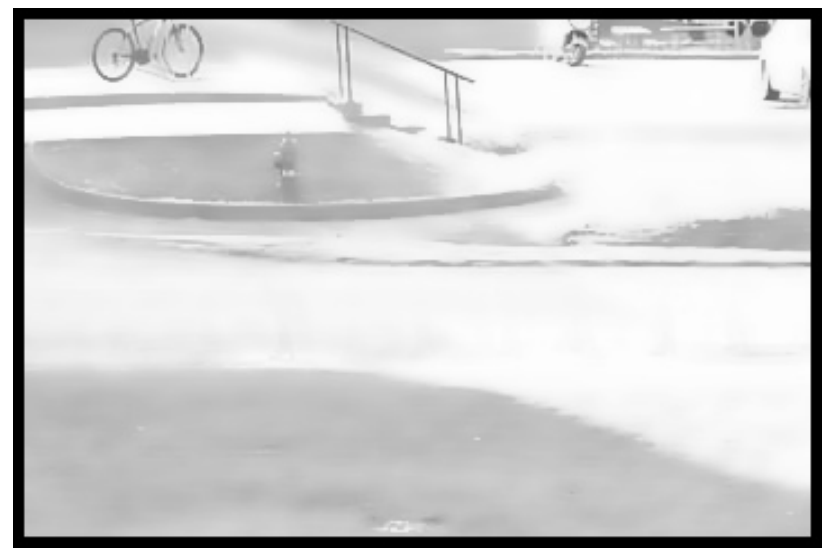

Figure 1. Smoothed memory $m_{n}^{*}(k)$.

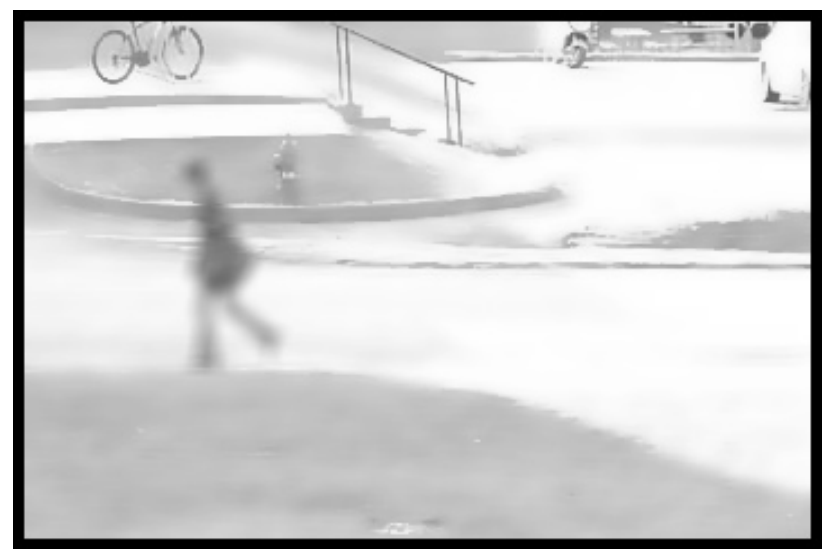

Figure 2. Image projection $I^{*}(k)$.

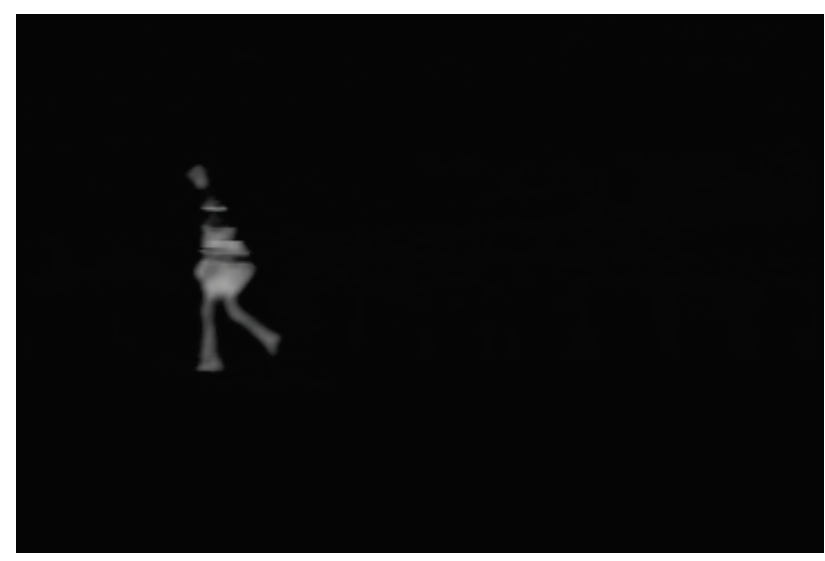

Figure 3. Background difference $D(k)$.

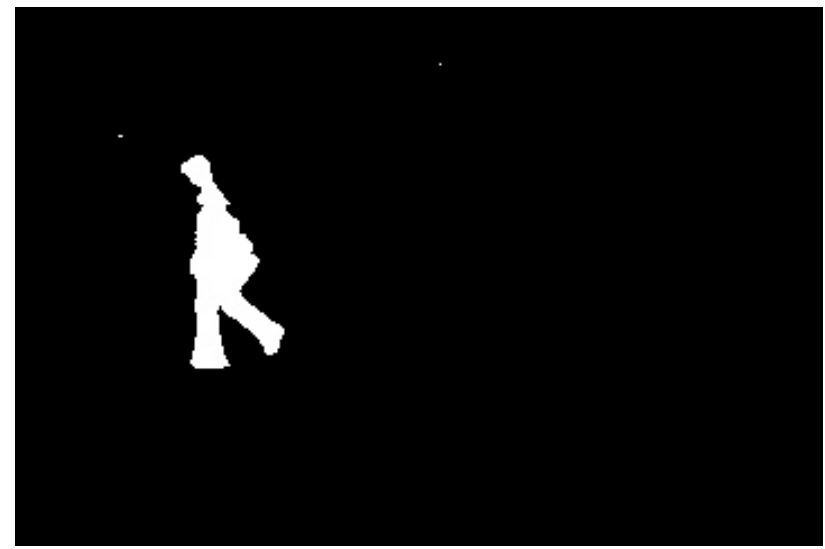

Figure 4. Binarized background difference $M(k)$.

\section{EXPERIMENTS}

In order to demonstrate the quality characteristics of the proposed approach we use the common benchmark videos from changedetection.net database (changedetection.net, 2014). Changedetection.net provide a realistic, camera-captured, diverse set of 31 videos ( 70,000 frames). They have been selected to cover a wide range of detection challenges and are representative of typical indoor and outdoor visual data captured today in surveillance, smart environment and video database scenarios. The 2014 dataset consist of different categories. We choose «baseline», «thermal», «bad weather», «low framerate» categories, because the proposed approach can be directly applicable to them in the presented straightforward form (regression model of background learning). Other «camera jitter», «shadow», «PTZ» (pan-tilt zoom) scenarios are not considered, because they require some special modification of the basic algorithm, we hope to explore them in the future. «Baseline» category represents a mixture of moderate challenges. Some videos have subtle background motion, others have isolated shadows, some have an abandoned object and others have pedestrians that stop for a short while and then move away. "Thermal» category includes videos that have been captured by far-infrared cameras. These videos contain typical thermal artifacts such as heat stamps (e.g., bright spots left on a seat after a person gets up and leaves), heat reflection on floors and windows, and camouflage effects, when a moving object has the same temperature as the surrounding regions. «Bad Weather» category includes outdoor videos captured in challenging winter weather conditions, i.e., snow storm, snow on the ground, fog. «Low Frame-Rate» category contains videos capture at varying frame-rates between $0.17 \mathrm{fps}$ and $1 \mathrm{fps}$. For «baseline» and «thermal» categories the results of proposed 
approach was compared to 37 other existed methods results available from change detection 2012 benchmark. For «Bad Weather», «Low Frame-Rate» categories the results of proposed approach was compared to 22 other existed methods results available from change detection 2014 benchmark.

The following methods were used for comparative analysis: SUBS (St-Charles, 2014), PAWCS (St-Charles, 2015), SOBS1 (L. Maddalena, 2012), SOBS2 (L. Maddalena, 2010), SOBS3 (L. Maddalena, 2008), PSP (A. Schick, 2012), S-360_1 (M. Sedky, 2011), MLBS (Jian Yao, 2007), GPGMM (T. S. F. Haines, 2012), SGMM (R. Heras, 2011), PBAS2 (M. Hofmann, 2012), KDE1 (A. Elgammal, 2000), BB (F. Porikli, 2005), CDPS (Francisco J. Hernandez, 2013), HIST (J. Zheng, 2006), CWD (M. De Gregorio, 2013), KNN (Z. Zivkovic, 2006), GMM1 (P. KaewTraKulPong, 2001), MAHAL (Y. Benezeth, 2010), CHEB1 (A. Morde, 2012), RMOG (S. Varadarajan, 2013), LSS (J-P Jodoin, 2012), GMM2 (Z. Zivkovic, 2004), ED (Y. Benezeth, 2010), GMM3 (D. Riahi, 2012), SGMM (Heras Evangelio, 2012), UBA (D. Park, 2009), PROST (F. Seidel, 2014), GMM4 (C. Stauffer, 1999) TUBI (L. Dar-Shyang, 2005), KDE2 (Y. Nonaka, 2012), KDE3 (S. Yoshinaga, 2013), QCHMD (O. Strauss, 2012), CHEB2 (A. Morde, 2012), FTSG (R. Wang, 2014), SS (Zhenkun, 2015), BWA (B. Wang, 2014), EFIC (G. Allebosch, 2015), S-360_2 (M.Sedky,2014), CWDH (M. De Gregorio, 2014), UBSS (H. Sajid, 2015), MSTBGM (Xiqun Lu, 2014).

Quantitative results for «baseline» category are shown in Tables 1, 2, 3. The following metrics are used: Re - Recall, SP (Specificity), FPR - False Positive Rate, FNR - False Negative Rate, PWC - Percentage of Wrong Classifications, F - FMeasure, PR - Precision, TP - True Positive, FP - False Positive, FN - False Negative, TN - True Negative, Rank - rank among all comparative methods in chosen category (38 or 22).

\begin{tabular}{|c|c|c|c|c|}
\hline DIFF & TP & FP & FN & TN \\
\hline pedestrians & 619693 & 122498 & 51169 & 67446609 \\
\hline PETS2006 & 2915070 & 295655 & 1913120 & 366417649 \\
\hline highway & 4728297 & 1220961 & 729692 & 85433508 \\
\hline office & 7932008 & 303147 & 710086 & 116235539 \\
\hline baseline & 16195068 & 1942261 & 3404067 & 635533305 \\
\hline
\end{tabular}

Table 1. Baseline category. Results for each video in this category.

\begin{tabular}{|l|c|c|c|c|c|c|c|}
\hline Method & RE & SP & FPR & FNR & PWC & F & PR \\
\hline SUBS & 0,95 & 0,99 & 0,002 & 0,05 & 0,35 & 0,95 & 0,94 \\
\hline PAWCS & 0,94 & 0,99 & 0,002 & 0,05 & 0,44 & 0,93 & 0,93 \\
\hline SOBS1 & 0,93 & 0,99 & 0,002 & 0,06 & 0,37 & 0,93 & 0,93 \\
\hline CDET & 0,97 & 0,99 & 0,002 & 0,02 & 0,35 & 0,94 & 0,92 \\
\hline SOBS2 & 0,93 & 0,99 & 0,002 & 0,06 & 0,39 & 0,92 & 0,92 \\
\hline SOBS3 & 0,91 & 0,99 & 0,002 & 0,08 & 0,43 & 0,92 & 0,93 \\
\hline GPRMF & 0,9 & 0,99 & 0,002 & 0,09 & 0,46 & 0,92 & 0,95 \\
\hline PSP & 0,93 & 0,99 & 0,002 & 0,06 & 0,41 & 0,92 & 0,96 \\
\hline S-360_1 & 0,96 & 0,99 & 0,003 & 0,03 & 0,42 & 0,93 & 0,90 \\
\hline MLBS & 0,84 & 0,99 & 0,001 & 0,15 & 0,89 & 0,90 & 0,96 \\
\hline DPGMM & 0,96 & 0,99 & 0,003 & 0,03 & 0,49 & 0,92 & 0,89 \\
\hline PBAS1 & 0,95 & 0,99 & 0,002 & 0,04 & 0,48 & 0,92 & 0,89 \\
\hline SGMM & 0,93 & 0,99 & 0,002 & 0,06 & 0,54 & 0,92 & 0,91 \\
\hline
\end{tabular}

\begin{tabular}{|l|l|l|l|l|l|l|l|} 
PBAS2 & 0,95 & 0,99 & 0,003 & 0,04 & 0,48 & 0,92 & 0,89 \\
\hline KDE1 & 0,89 & 0,99 & 0,002 & 0,10 & 0,54 & 0,90 & 0,92 \\
\hline BB & 0,73 & 0,99 & 0,001 & 0,26 & 0,90 & 0,82 & 0,96 \\
\hline CDPS & 0,94 & 0,99 & 0,003 & 0,05 & 0,62 & 0,92 & 0,89 \\
\hline HIST & 0,87 & 0,99 & 0,002 & 0,12 & 0,66 & 0,90 & 0,92 \\
\hline CWD & 0,89 & 0,99 & 0,002 & 0,10 & 0,66 & 0,90 & 0,91 \\
\hline KNN & 0,79 & 0,99 & 0,002 & 0,20 & 1,28 & 0,84 & 0,92 \\
\hline GMM1 & 0,58 & 0,99 & 0,001 & 0,41 & 1,93 & 0,71 & 0,95 \\
\hline MAHAL & 0,88 & 0,99 & 0,003 & 0,11 & 0,72 & 0,89 & 0,90 \\
\hline CHEB1 & 0,82 & 0,99 & 0,003 & 0,17 & 0,83 & 0,86 & 0,91 \\
\hline RMOG & 0,70 & 0,99 & 0,001 & 0,29 & 1,59 & 0,78 & 0,91 \\
\hline LSS & 0,97 & 0,98 & 0,013 & 0,02 & 1,33 & 0,84 & 0,75 \\
\hline GMM2 & 0,80 & 0,99 & 0,002 & 0,19 & 1,32 & 0,83 & 0,89 \\
\hline ED & 0,83 & 0,99 & 0,004 & 0,16 & 1,02 & 0,87 & 0,91 \\
\hline GMM3 & 0,66 & 0,99 & 0,002 & 0,33 & 1,53 & 0,75 & 0,91 \\
\hline SGMM & 0,86 & 0,99 & 0,005 & 0,13 & 1,24 & 0,85 & 0,85 \\
\hline DIFF* & $\mathbf{0 , 8 2}$ & $\mathbf{0 , 9 9}$ & $\mathbf{0 , 0 0 4}$ & $\mathbf{0 , 1 7}$ & $\mathbf{0 , 9 4}$ & $\mathbf{0 , 8 4}$ & $\mathbf{0 , 8 7}$ \\
\hline UBA & 0,90 & 0,99 & 0,008 & 0,09 & 1,01 & 0,81 & 0,74 \\
\hline PROST & 0,84 & 0,99 & 0,006 & 0,15 & 1,15 & 0,82 & 0,81 \\
\hline GMM4 & 0,81 & 0,99 & 0,005 & 0,18 & 1,53 & 0,82 & 0,84 \\
\hline TUBI & 0,89 & 0,98 & 0,015 & 0,10 & 2,08 & 0,76 & 0,67 \\
\hline KDE2 & 0,74 & 0,99 & 0,004 & 0,25 & 1,80 & 0,73 & 0,79 \\
\hline KDE3 & 0,75 & 0,99 & 0,00 & 0,24 & 1,91 & 0,75 & 0,78 \\
\hline QCHMD & 0,70 & 0,99 & 0,007 & 0,29 & 2,21 & 0,66 & 0,70 \\
\hline
\end{tabular}

Table 2. Baseline category. DIFF* - proposed method.

\begin{tabular}{|c|c|c|c|c|c|c|c|c|}
\cline { 2 - 9 } \multicolumn{1}{c|}{} & \multicolumn{7}{c|}{ Ranks among 38 methods } \\
\cline { 2 - 9 } \multicolumn{1}{c|}{} & RE & SP & FPR & FNR & PWC & F & PR & Overal \\
\hline DIFF & 26 & 29 & 29 & 26 & 22 & 24 & 28 & $30 / 38$ \\
\hline
\end{tabular}

Table 3. Baseline category. Rank for each metric for proposed method presented.

Results for «Thermal» category is shown in Table 4, 5, 6.

\begin{tabular}{|c|c|c|c|c|c|c|c|c|}
\hline DIFF & \multicolumn{3}{|c|}{$\mathbf{T P}$} & FP & \multicolumn{2}{|c|}{ FN } & \multicolumn{2}{|r|}{ TN } \\
\hline corridor & \multicolumn{3}{|c|}{10667192} & 7675519 & \multicolumn{2}{|c|}{1672965} & \multicolumn{2}{|c|}{352311506} \\
\hline dining & \multicolumn{3}{|c|}{16264143} & 4775408 & \multicolumn{2}{|c|}{3200154} & \multicolumn{2}{|c|}{202408859} \\
\hline lakeSide & \multicolumn{3}{|c|}{5035690} & 26973588 & \multicolumn{2}{|c|}{2937391} & \multicolumn{2}{|c|}{381184826} \\
\hline library & \multicolumn{3}{|c|}{58508839} & 3889517 & \multicolumn{2}{|c|}{3452426} & \multicolumn{2}{|c|}{255590998} \\
\hline park & \multicolumn{3}{|c|}{384658} & 48641 & \multicolumn{2}{|c|}{330479} & \multicolumn{2}{|c|}{34446499} \\
\hline thermal & \multicolumn{3}{|c|}{90860522} & 43362673 & \multicolumn{2}{|c|}{11593415} & \multicolumn{2}{|c|}{1225942688} \\
\hline \multicolumn{9}{|c|}{$\begin{array}{l}\text { Table 4. Thermal category. Results for each video in this } \\
\text { category. }\end{array}$} \\
\hline Method & & $\mathbf{R E}$ & SP & FPR & FNR & PWC & $\mathbf{F}$ & PR \\
\hline DIFF & & 0,76 & 0,98 & 0,025 & 0,23 & 3,31 & 0, & 0,67 \\
\hline \multicolumn{9}{|c|}{ Table 5. Thermal category. } \\
\hline & \multicolumn{8}{|c|}{ Ranks among 38 methods } \\
\hline & & SP & FPR & FNR & PWC & $\mathbf{F}$ & PR & Overall \\
\hline
\end{tabular}




\section{\begin{tabular}{l|l|l|l|l|l|l|l|l|}
\hline DIFF & 7 & 37 & 37 & 7 & 24 & 24 & 37 & $32 / 38$ \\
\hline
\end{tabular}}

Table 6. Thermal category. Rank for each metric for proposed method presented.

Results for «Bad Weather» category is shown in Table 7, 8, 9 .

\begin{tabular}{|c|c|c|c|c|}
\hline DIFF & TP & FP & FN & TN \\
\hline blizzard & 8105421 & 114550 & 4151098 & 1038823675 \\
\hline skating & 11950348 & 2149566 & 2823328 & 281628841 \\
\hline snowFall & 5100041 & 931585 & 2656794 & 975209889 \\
\hline wetSnow & 3439753 & 694655 & 2575110 & 460144956 \\
\hline weather & 28595563 & 3890356 & 12206330 & 2755807361 \\
\hline
\end{tabular}

Table 7. Bad Weather category. Results for each video in this category.

\begin{tabular}{|l|c|c|c|c|c|c|c|}
\hline Methods & RE & SP & FPR & FNR & PWC & F & PR \\
\hline DIFF & 0,68 & 0,99 & 0,002 & 0,32 & 0,78 & 0,76 & 0,88 \\
\hline
\end{tabular}

Table 8 . Bad Weather category.

\begin{tabular}{|c|c|c|c|c|c|c|c|c|}
\cline { 2 - 8 } \multicolumn{1}{c|}{} & \multicolumn{7}{c|}{ Ranks among 22 methods } \\
\cline { 2 - 9 } \multicolumn{1}{c|}{} & RE & SP & FPR & FNR & PWC & F & PR & Overall \\
\hline DIFF & 12 & 16 & 16 & 12 & 14 & 9 & 11 & $15 / 22$ \\
\hline
\end{tabular}

Table 9. Bad Weather category. Rank for each metric for proposed method presented.

Results for «Low Frame-Rate» category is shown in Table 10, 11,12 .

\begin{tabular}{|c|c|c|c|c|}
\hline DIFF & TP & FP & FN & TN \\
\hline port & 19425 & 83230 & 26366 & 153835893 \\
\hline tram & 290806 & 96304 & 64765 & 12357342 \\
\hline tunnel & 1283430 & 135444 & 3702953 & 176739284 \\
\hline turn & 1355782 & 259399 & 450049 & 22203801 \\
\hline low fr. r. & 2949443 & 574377 & 4244133 & 365136320 \\
\hline
\end{tabular}

Table 10. Low Frame-Rate category. Results for each video in this category.

\begin{tabular}{|l|c|c|c|c|c|c|c|}
\hline Methods & RE & SP & FPR & FNR & PWC & F & PR \\
\hline DIFF & 0,56 & 0,99 & 0,005 & 0,43 & 1,59 & 0,55 & 0,67 \\
\hline
\end{tabular}

Table 11. Low Frame-Rate category.

\begin{tabular}{|c|c|c|c|c|c|c|c|c|}
\cline { 2 - 9 } \multicolumn{1}{c|}{} & \multicolumn{7}{c|}{ Ranks among 22 methods } \\
\cline { 2 - 9 } & RE & SP & FPR & FNR & PWC & F & PR & Overall \\
\hline DIFF & 18 & 10 & 10 & 18 & 13 & 8 & 8 & $13 / 22$ \\
\hline
\end{tabular}

Table 12. Low Frame-Rate category. Rank for each metric for proposed method presented.

From conducted experiments we conclude that the proposed approach can be used for background modelling, performs better than several well-known methods or is comparative to them in case of different shooting conditions.

\section{CONCLUSION}

\section{REFERENCES}

Ahonen, T., Hadid, A., Pietikainen, M., 2004. Face recognition with local binary patterns. ECCV, pp 469-481
G. Allebosch, D. Van Hamme, F. Deboeverie, P. Veelaert and W. Philips "Edge based foreground background segmentation with interior/exterior classification". To be published in proceedings of VISAPP 2015

Y. Benezeth, P.-M. Jodoin, B. Emile, H. Laurent, and C. Rosenberger. Comparative study of background subtraction algorithms.J. of Elec. Imaging, 19(3):1-12, 2010.

St-Charles, P.-L., Bilodeau, G.-A., Bergevin, R., "SuBSENSE : A Universal Change Detection Method with Local Adaptive Sensitivity". Accepted for IEEE Transactions on Image Processing, Nov. 2014.

St-Charles, P.-L., Bilodeau, G.-A., Bergevin, R., "SuBSENSE: A Universal Change Detection Method with Local Adaptive Sensitivity". Accepted for IEEE Transactions on Image Processing, Nov. 2014

P.-L. St-Charles, G.-A. Bilodeau, R. Bergevin, "A Self-Adjusting Approach to Change Detection Based on Background Word Consensus" in IEEE Winter Conference on Applications of Computer Vision (WACV). Big Island, Hawaii, USA, Jan. 6-9, 2015.

Coifman, R., Lafon, S., Maggioni, M., Keller, Y., D Szlam, A, Warner, F., Zucker, S. Geometries of sensor outputs, inference and information processing // Storage and Re-trieval for Image and Video Databases, edited by Intelligent Integrated Microsystems - 2006. - Vol. 6232 - P. 623209.

Coifman, R., Lafon, S. Diffusion maps // Applied and Computational Harmonic Analysis. - 2006. - Vol. 21(1). - P. 5 30 .

Lee, Dar-Shyang. "Effective Gaussian mixture learning for video background subtraction." Pattern Analysis and Machine Intelligence, IEEE Transactions on 27.5 (2005): 827-832.

Dong Liang, Shun'ichi Kaneko, "Improvements and Experiments of a Compact Statistical Background Model", arXiv:1405.6275, 2014

A. Elgammal, D. Harwood, and L. Davis, "Non-parametric model for background subtraction," in Proc. Eur. Conf. on Computer Vision, Lect. Notes Comput. Sci. 1843, 751-767 2000 .

Francisco J. Hernandez-Lopez and Mariano Rivera. Change Detection by Probabilistic Segmentation from Monocular View. Machine Vision and Applications, pages 1-21, 2013.

M. De Gregorio and M. Giordano "A WiSARD-based approach to CDnet" 1-st BRICS Countries Congress (BRICS-CCI) - 2013.

M. De Gregorio and M. Giordano "Change Detection with Weightless Neural Networks", in proc of IEEE Workshop on Change Detection, 2014.

T. S. F. Haines and T. Xiang "Background Subtraction with Dirichlet Processes" ECCV 2012

R. Heras and T. Sikora "Complementary Background Models for the Detection of Static and Moving Objects in Crowded Environments", in 8th Proceedings of the IEEE International Conference on Advanced Video and Signal-Based Surveillance (AVSS), 2011. 
Heras Evangelio, R. and Pätzold, M. and Sikora, T. "Splitting Gaussians in Mixture Models", Proceedings of the 9th IEEE International Conference on Advanced Video and Signal-Based Surveillance, 2012.

V. Gorbatsevich, D. Pronin, Y. Vizilter Diffusion maps fast algorithm implementation // TCVS-2014 theses - 2014. In Russian.

N. Goyette, P.-M. Jodoin, F. Porikli, J. Konrad, and P. Ishwar, changedetection.net: A new change detection benchmark dataset, in Proc. IEEE Workshop on Change Detection (CDW-2012) at CVPR-2012, Providence, RI, 16-21 Jun., 2012.

M. Hofmann, P. Tiefenbacher, G. Rigoll "Background Segmentation with Feedback: The Pixel-Based Adaptive Segmenter", in proc. of IEEE Workshop on Change Detection, 2012.

J-P Jodoin, G-A Bilodeau, N Saunier "Background subtraction based on Local Shape", arXiv:1204.6326v1 , 27 Apr 2012.

Jian Yao and Jean-Marc Odobez, Multi-Layer Background Subtraction Based on Color and Texture. Visual Surveillance workshop (CVPR-VS), Minneapolis, June 2007.

P. KaewTraKulPong and R. Bowden, "An Improved Adaptive Background Mixture Model for Real-time Tracking with Shadow Detection", in proc. of Workshop on Advanced Video Based Surveillance Systems, 2001

D. Kit, B. T. Sullivan, and D. H. Ballard. Novelty detection using growing neural gas for visuo-spatial memory. In IROS, pages 1194-1200. IEEE, 2011.

Lafon, S. Diffusion maps and geometric harmonics // $\mathrm{PhD}$ thesis. - Yale University, Dept of Mathematics \& Applied Mathematics. -2004 .

L. Maddalena, A. Petrosino, A Self-Organizing Approach to Background Subtraction for Visual Surveillance Applications, IEEE Transactions on Image Processing, Vol. 17, no.7, 2008, p1168-1177.

L. Maddalena, A. Petrosino, A Fuzzy Spatial Coherence-based Approach to Background/ Foreground Separation for Moving Object Detection, Neural Computing and Applications, Springer London, Vol. 19, pp. 179-186, 2010.

L. Maddalena, A. Petrosino, "The SOBS algorithm: what are the limits", in proc of IEEE Workshop on Change Detection, CVPR 2012.

A. Morde, X. Ma, S. Guler [IntuVision] "Learning a background model for change detection", in proc. of IEEE Workshop on Change Detection, 2012.

Y. Nonaka, A. Shimada, H. Nagahara, R. Taniguchi "Evaluation Report of Integrated Background Modeling Based on Spatiotemporal Features", in proc. of IEEE Workshop on Change Detection, 2012

D. Park, H. Byun, Object-wise multilayer background ordering for pubic area surveillance", in IEEE International Conference on AVSS, 2009
F. Porikli and O. Tuzel. "Bayesian background modeling for foreground detection" in proc. of ACM Visual Surveillance and Sensor Network, 2005.

Riahi, D., St-Onge, P.L., Bilodeau, G.A. (2012). RECTGAUSSTex: Block-based Background Subtraction, Technical Report, École Polytechnique de Montréal, EPM-RT-2012-03.

Satoshi Yoshinaga, Atsushi Shimada, Hajime Nagahara, Rinichiro Taniguchi, Background Model Based on Intensity Change Similarity Among Pixels, the 19th Japan-Korea Joint Workshop on Frontiers of Computer Vision, pp.276-280, 2013.01.

A. Schick, M. Bäuml, R. Stiefelhagen "Improving Foreground Segmentations with Probabilistic Superpixel Markov Random Fields", in proc of IEEE Workshop on Change Detection, 2012.

SEDKY Mohamed Hamed Ismail, MONIRI Mansour and CHIBELUSHI Claude Chilufya "Object Segmentation Using Full-Spectrum Matching of Albedo Derived from Colour Images" UK patent application no. 0822953.6 16.12.2008 GB, 2008, PCT patent application international application no. PCT/GB2009/002829, EP2374109, 2009, US patent no. 2374109 12.10.2011 US, 2011.

M. Sedky, M. Moniri and C. C. Chibelushi "Spectral-360: A physical-based technique for change detection", in proc of IEEE Workshop on Change Detection, 2014.

F. Seidel, C. Hage, M. Kleinsteuber "pROST - A Smoothed Lpnorm Robust Online Subspace Tracking Method for Real time Background Subtraction in Video". In Machine Vision and Applications, 25(5):1227-1240, 2014.

C. Stauffer and W. E. L. Grimson, "Adaptive background mixture models for real-time tracking," in Proc. Int. Conf. on Computer Vision and Pattern Recognition, Vol. 2, IEEE, Piscataway, NJ (1999).

O. Strauss, D. Sidibé, W. Puech "Quasi-continuous histogram based motion detection", Technical Report, LE2I, 2012.

Varadarajan, S.; Miller, P.; Huiyu Zhou, "Spatial mixture of Gaussians for dynamic background modelling," Advanced Video and Signal Based Surveillance (AVSS), 2013 10th IEEE International Conference on , pp.63,68, 27-30 Aug. 2013.

Vishnyakov, B., Vizilter, Y., Knyaz V. Spectrum-Based Object Detection And Tracking Technique For Digital Video Surveillance // ISPRS Archives - XXXIX-B3 - 2012. - P. 579583.

Vishnyakov, B., Gorbatsevich, V., Sidyakin, S., Malin, I., Egorov, A Fast moving objects detection using iLBP background model // Int. Arch. Photogramm. Remote Sens. Spatial Inf. Sci., XL-3 - 2014 - P. 347-350.

Vizilter Y., Gorbatsevich V., Rubis A., Vygolov O. Image comparison using shape matching diffusion correlation morthology // Computer Optics, 2013, Vol. 37, 1. In Russian.

R. Wang, F. Bunyak, G. Seetharaman and K. Palaniappan "Static and Moving Object Detection Using Flux Tensor with Split Gaussian Models", in proc of IEEE Workshop on Change Detection, 2014. 
Y. Wang, P.-M. Jodoin, F. Porikli, J. Konrad, Y. Benezeth, and P. Ishwar, CDnet 2014: An Expanded Change Detection Benchmark Dataset, in Proc. IEEE Workshop on Change Detection (CDW-2014) at CVPR-2014, pp. 387-394. 2014.

B. Wang and P. Dudek "A Fast Self-tuning Background Subtraction Algorithm", in proc of IEEE Workshop on Change Detection, 2014.

Xiqun $\mathrm{Lu}$ "A multiscale spatio-temporal background model for motion detection", ICIP 2014

Yin, Baocai; Zhang, Jing; Wang, Zengfu: 'Background segmentation of dynamic scenes based on dual model', IET Computer Vision, 2014, DOI: 10.1049/iet-cvi.2013.0319 IET Digital Library, http://digitallibrary.theiet.org/content/journals/10.1049/iet-cvi.2013.0319.

J. Zheng, Y. Wang, N.L. Nihan, and M.E. Hallenbeck. Extracting roadway background image : Mode-based approach. Transportation Research Record : Journal of the Transportation Research Board, 1944(-1) :82-88, 2006.

Zhenkun huang Ruimin $\mathrm{Hu}$ and Shihong Chen, paper has been submitted to CVPR2015.

Z. Zivkovic, "Improved adaptive Gaussian mixture model for back-ground subtraction," in Proc. Int. Conf. Pattern Recognition, pp. 28-31, IEEE, Piscataway, NJ 2004.

Z. Zivkovic, F. van der Heijden "Efficient adaptive density estimation per image pixel for the task of background subtraction", Pattern Recognition Letters, vol. 27, no. 7, pages 773-780, 2006.

\section{APPENDIX}

\section{C++ - LIKE SOURCE CODE FOR OUR COMPLEX LBP DIFFISUION FILTERING}

Pseudo-code of Diffusion filtering and processing that is used in Diffusion Background Model.

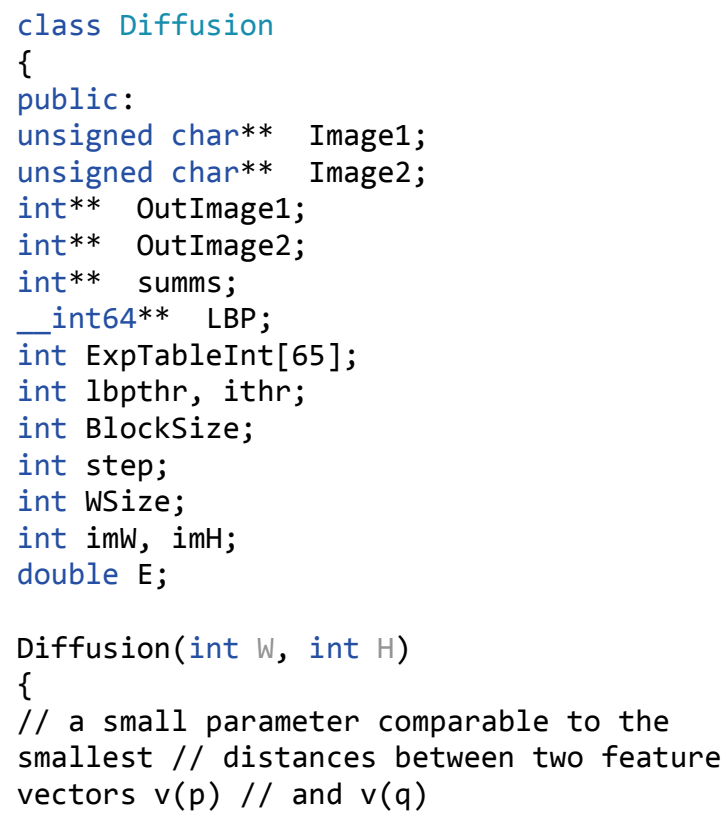

$\mathrm{E}=10$

// LBP threshold

lbpthr = 10;

// Intensity threshold

ithr = 50;

// Half of the sliding window (neighbourhood)

BlockSize $=5$;

WSize = BlockSize; step $=1$;

// Pre-calculating table values for exponent for (int $i=0$; $i<65$; $i++$ )

ExpTableInt [i] = (int) $(\exp (-\mathrm{i} / \mathrm{E}) * 1024)$;

$i m W=W$; $i m H=H$;

Image1 = new unsigned $\operatorname{char} *[\mathrm{H}]$;

Image 2 = new unsigned char* $[\mathrm{H}]$;

$\mathrm{LBP}=$ new _int $64 *[\mathrm{H}]$;

$\operatorname{LBP}[\theta]=$ new $\ldots$ int $64\left[\mathrm{~W}^{*} \mathrm{H}\right]$;

summs $=$ new int $*[\mathrm{H}]$;

summs $[0]=$ new int $\left[\mathrm{H}^{*} \mathrm{~W}\right]$;

OutImage $1=$ new int $*[\mathrm{H}]$;

OutImage 10$]=$ new int $\left[\mathrm{H}^{*} \mathrm{~W}\right]$;

OutImage $2=$ new int $*[\mathrm{H}]$;

OutImage2[0] = new int $\left[\mathrm{H}^{*} \mathrm{~W}\right]$;

for (int $i=1 ; i<H$; $i++$ )

\{

$\operatorname{LBP}[i]=\& \operatorname{LBP}[\theta][i * W]$;

OutImage1 $[i]=$ \&OutImage1 $[\theta][i * i m W]$;

OutImage $2[i]=$ \&OutImage $2[0][i * i m W] ;$

summs $[i]=\&$ summs $[\theta][i * i m W]$;

\}

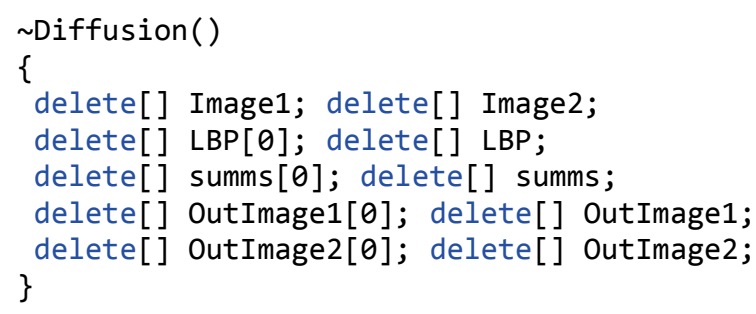

//diffusion filtering of image 1 by itself void Process(unsigned char* image1)

\{

for (int $i=0$; $i<i m H ; i++$ )

Image1 $[i]=$ \&image1 $[i * i m W]$;

BuildLBPMap( );

\}

//diffusion projection of image 2 on the form of //image1

void Project(unsigned char* image1, unsigned

\{ char* image 2 )

for (int $i=0$; $i<i m H ; i++$ )

\{

Image1 $[i]=$ \&image1 $[i * i m W] ;$

\}

// LBP pre-calculation with lbpthr threshold void BuildLBPMap()

\{ 
